\title{
Effects of Curing Modes on the Microhardness of Resin-Modified Glass lonomer Cements
}

\section{Utjecaj načina polimerizacije na mikrotvrdoću staklenoionomernih cemenata modificiranih smolom}

\author{
${ }^{1}$ Health Center, Runjaninova 4, Zagreb \\ Dom zdravlja, Runjaninova 4, Zagreb \\ 2 Department of Endodontics and Restorative Dental Medicine School of Dental Medicine University of Zagreb, Gundulićeva 5, Zagreb \\ Zavod za endodonciju i restaurativnu stomatologiju Stomatološkog fakulteta Sveučilišta u Zagrebu, Zagreb \\ 3 Institute of Physics, Bijenička 46, Zagreb \\ Institut za fiziku, Zagreb \\ ${ }^{4}$ Private Dental Office, Sisak, Croatia \\ Privatna ordinacija dentalne medicine, Sisak
}

\section{Abstract}

Objective: This study evaluated the effects of curing modes on surface microhardness of visible lightcured resin-modified glass ionomer cements (VLC RMGIC) and a giomer after different storage periods in comparison to auto-cured resin-modified glass ionomer cements (AC RMGIC). Materials and Methods: The following materials were used: VLC RMIC: Fuji II LC Improved, Photac Fil Quick Aplicap, AC RMGIC: Fuji Plus, Fuji VIII and Giomer: Beautifil II. The measurements of microhardness were performed using a Vickers test (100 g loads were applied for $10 \mathrm{~s}$ ) in the following time intervals: immediately after the recommended cure and after 1,7 and 14 days of immersion in distilled water. Five samples $(d=4 \mathrm{~mm}, \mathrm{~h}=2 \mathrm{~mm})$ were prepared for each combination of curing mode and tested material. Results: After 14 days, an improvement of microhardness was evident in all tested materials. The full factorial ANOVA identified a highly significant $(p<0.001)$ effect of the factors "material", "time" and "curing mode ("low", "soft", ,high”) for the light-cured materials Beautifil II, Fuji II LC and Photac Fil Quick. There was a statistically significant difference in the microhardness between different material types (Beautifil II>Fuji II LC>Photac Fil Quick>Fuji Plus $>$ Fuji VIII) and curing modes (low $<$ soft $<$ high). Conclusions: Material type had the greatest impact on microhardness, followed by the factor of time, while curing modes showed a considerably smaller influence on microhardness of the lightcured materials.
Received: September 30, 2018 Accepted: December 1, 2018

Address for correspondence Professor Katica Prskalo, Ph.D. University of Zagreb School of Dental Medicine Department of Endodontics and Restorative Dentistry Gundulićeva 5, 10000 Zagreb, Croatia.

tel: 014802126

prskalo@sfzg.hr

Key words

Dental Materials; Light-Curing of Dental Adhesives; Glass lonomer Cements; Hardness Tests

\section{Introduction}

After almost 45 years of clinical use glass ionomer cements (GICs) have undergone some changes aiming to improve their properties, handling characteristics, efficacy and longevity of restorations. One of important changes is hydrophilic resin monomer incorporation to GIC, resulting in resin-modified GIC (RMGIC) (1).

This modification was an attempt to overcome moisture sensitivity and low physical properties of conventional GIC while still maintaining unique properties of GIC such as chemical adhesion to tooth structure and fluoride release (2).

Basically, RMGICs consist of ion-leachable glass, watersoluble polymeric acids, polymerizable organic monomers with appropriate initiation system and water (3). Nowadays the available RMGIC products contain different combinations of methacrylate monomers, among which 2-hydroxyethylmethacrylate is (HEMA) the most commonly used (4-7).

\section{Uvod}

Tijekom otprilike 45 godina kliničke uporabe, staklenoionomerni cementi (SIC) podvrgnuti su određenim promjenama radi poboljšanja njihovih svojstava, lakšeg rukovanja, učinkovitosti i trajnosti restauracija. Jedna od važnijih promjena jest uvođenje hidrofilnoga smolastog monomera u SIC, što je rezultiralo SIC-om (SMSIC-om) modificiranim smolom (1).

Tom modifikacijom pokušao se riješiti problem osjetljivosti na vlagu i loša fizikalna svojstva konvencionalnog SICa, uz istodobno zadržavanje njegovih jedinstvenih svojstava poput kemijske adhezije za zub i otpuštanja fluorida (1).

U osnovi se SMSIC-ovi sastoje od topljivih čestica stakla, polimernih kiselina topljivih u vodi, polimerizirajućih organskih monomera s odgovarajućim inicijatorom polimerizacije te vode (2). Danas dostupni SMSIC-ovi sadržavaju različite kombinacije metakrilnih monomera, među kojima se najčešće koristi 2-hidroksietilmetakrilat (HEMA) (4-7). 
Resin monomer incorporation modifies the chemistry of the GIC setting reaction (8). The fundamental acid-base reaction begins as soon as the powder (base) and liquid (acid) are combined, forming the network of polysalts. This reaction lasts approximately several minutes, although further maturation continues over extended times (9). Acid-base reaction is supplemented by free-radical mediated polymerization of methacrylate monomers, whereas both reactions occur concurrently, if not disturbed. Monomer polymerization can be induced chemically or photo-chemically depending on the initiator system used. Thus, RMGICs available on the market can be designated as dual cure (acid-base reaction + monomer light-cure or acid-base reaction + monomer autocure) or triple cure (acid-base reaction + monomer light-cure + monomer auto-cure) materials.

The set of RMGIC has a complicated structure of interpenetrating networks of poly (HEMA) and polyacrylate salts in which the unreacted (10), remaining parts of glass particles are embedded.

At an early stage of the setting process, there is a competitive nature of network-forming reactions $(11,12)$ and a sensitive balance exists between these two processes (12).

The setting reaction of light-cured RMGIC (VLC RMGIC) involves a rapid polymerization reaction of the resinous component and a slower acid-base reaction, both of which mutually interact (13). Visible light command set of RMGIC has an advantage in clinical procedure, because polymerized monomer immediately protects the acid-base reaction from the problems of water balance and stabilizes the setting cement thus allowing the continuation of clinical procedure.

It is important to distinguish RMGICs from the materials that resemble GICs, namely polyacid modified resin composite (3) and giomers that are compositionally more similar to light cured resin composites. Giomers contain surface reaction type of pre-reacted glass-ionomer (S-PRG) fillers (14) obtained by reacting acid-reactive fluoride-containing glass with polyacids in the presence of water (15) and are capable of fluoride release/recharge in a wet environment.

RMGICs represent an established class of restorative materials with good clinical performance. Nevertheless, potential cytotoxicity may occur from the presence of the residual monomer (16). Palmer et al. (17) investigated HEMA release from Fuji Lining LC, Vitremer, Vitrebond and Fuji II LC and found different material behavior with regards to the effect of curing time.

Apart from the potential cytotoxicity, it is very important to achieve an adequate polymerization of the resinous component in order to ensure basic mechanical properties and longevity of restorations (18). Polymerization effectiveness is influenced by the type of light curing unit (LCU), its irradiance and exposure time (18).

Nowadays, different types of LCUs are being used for the polymerization of light activated resinous materials (20), and halogen and light emitting diode light curing units (LED LCU) are most commonly used „third generation” of LED LCUs which generates multiple wavelengths The "third generation" of LED LCUs which generates multiple wavelengths
Uvođenje smolastog monomera modificira kemijski proces SIC-ove reakcije stvrdnjivanja (8). Osnovna kiselo-lužnata reakcija počinje čim se prah (lužina) i tekućina (kiselina) povežu, formirajući mrežu polisoli. Ta reakcija traje nekoliko minuta, iako se maturacija nastavlja (9). Kiselo-lužnata reakcija dopunjena je polimerizacijom metakrilatnih monomera posredovanom slobodnim radikalima, a obje reakcije događaju se istodobno, ako nisu ometane. Polimerizacija monomera može biti inducirana kemijski ili fotokemijski, ovisno o primijenjenom inicijatorskom sustavu. Stoga SMSIC-ovi koji su dostupni na tržištu mogu biti dvostruko stvrdnjavajući (kiselo-lužnata reakcija + svjetlosna polimerizacija monomera ili kiselo-lužnata reakcija + samostvrdnjivanje monomera) ili trostruko stvrdnjavajući (kiselo-lužnata reakcija + svjetlosna polimerizacija monomera + samostvrdnjivanje monomera).

Stvrdnuti SMSIC ima složenu strukturu interpenetriranih mreža poli(HEMA) i poliakrilatnih soli u kojima su smješteni nereagirani (10) preostali dijelovi čestica stakla.

U ranoj fazi procesa stvrdnjivanja prisutan je kompetitivni odnos između reakcija stvaranja mreža $(11,12)$ i postoji osjetljiva ravnoteža između tih dvaju procesa (12).

Reakcija stvrdnjivanja svjetlom polimerizirajućih SMSIC-a (SP SMSIC) uključuje brzu reakciju polimerizacije smolaste komponente i sporiju kiselo-lužnatu reakciju, a obje uzajamno djeluju (13). Svjetlosna polimerizacija SMSIC-a ima prednost $\mathrm{u}$ kliničkom radu, zato što polimerizirani monomer odmah štiti kiselo-lužnatu reakciju od problema s ravnotežom vode i stabilizira stvrdnjavajući cement, što omogućuje nastavak kliničkog postupka.

Važno je razlikovati SMSIC-ove od materijala koji sliče SIC-ovima, odnosno od polikiselinom modificiranih kompozitnih smola (2) i giomera koji su prema sastavu više slični svjetlom polimerizirajućim smolastim kompozitima. Giomeri sadržavaju površinski tip već reagiranih staklenoionomernih (S-PRG) čestica punila (14) dobivenih reakcijom reaktivnoga fluoridnog stakla s polikiselinama uz prisutnost vode (15) te mogu otpuštati/primati fluoride u vlažnim uvjetima.

SMSIC-ovi su dobro poznati restaurativni materijali $s$ dobrim kliničkim učincima. Unatoč tomu može se pojaviti citotoksičnost zbog rezidualnog monomera (16). Palmer i suradnici (17) istraživali su otpuštanje HEMA-e iz Fuji Lining LC-a, Vitremera, Vitrebonda i Fuji II LC-a te otkrili različito ponašanje materijala u odnosu prema trajanju polimerizacije.

Uz potencijalnu citotoksičnost, iznimno je važno postići odgovarajuću polimerizaciju smolaste komponente kako bi se postigla osnovna mehanička svojstva i dugotrajnost restauracije (18). Na učinkovitost polimerizacije utječu vrsta polimerizacijskog uređaja (PU), snaga i valna duljina zračenja te trajanje polimerizacije (18).

Danas se različiti tipovi PU-a koriste za svjetlosnu polimerizaciju smolastih materijala (20), a najčešći su halogeni polimerizacijski uređaji i uređaji temeljeni na svjetlećim diodama (LED). Posebno je učinkovita tré́a generacija LED uređaja koji generiraju višestruke valne duljine, poprilično učinkovite za polimerizaciju bilo koje vrste dentalnih restaurativnih materijala (20). 
is particularly effective for polymerization of any type of dental restorative material (20).

Multiple studies have compared the effectiveness of the halogen and LED LCU with similar $\left(600 \mathrm{~mW} / \mathrm{cm}^{2}\right)(21)$, or different light intensities (halogen $350 \mathrm{~mW} / \mathrm{cm}^{2}$, LED 1400 $\mathrm{mW} / \mathrm{cm}^{2}$, LED $\left.1100 \mathrm{~mW} / \mathrm{cm}^{2}\right)$ (22), and with different light intensity and exposure time (halogen $700 \mathrm{~mW} / \mathrm{cm}^{2}$, LED $500 \mathrm{~mW} / \mathrm{cm}^{2} ; 20 \mathrm{~s}$ and $\left.40 \mathrm{~s}\right)$ on the microhardness $(\mathrm{MH})$ of RMGIC (23). Microhardness (MH) value was dependent on the light used to cure them $(21,22)$. In relation to the curing time, similar $\mathrm{MH}$ results were obtained with halogen and LED $40 \mathrm{~s}$ time exposure, while a significant difference was found between halogen $40 \mathrm{~s}$ and LED $20 \mathrm{~s}$ (23). Parisay et al. (24) also compared halogen and LED curing units with different light intensity (halogen $600 \mathrm{~mW} / \mathrm{cm}^{2}$, LED $700 \mathrm{~mW} /$ $\left.\mathrm{cm}^{2}\right)$, and exposure time (20 s, $\left.30 \mathrm{~s}, 40 \mathrm{~s}\right)$ on top and bottom $\mathrm{MH}$. The top surface $\mathrm{MH}$ was higher in all experimental groups. An increase in both the top and the bottom $\mathrm{MH}$ was observed when exposure time was increased. In the halogen group, there were no significant differences between 20, 30 and $40 \mathrm{~s}$, while in the LED group, $40 \mathrm{~s}$ curing yielded significantly better results than 20 and $30 \mathrm{~s}$.

Also, there are studies that compared $\mathrm{MH}$ of resin-modified versus conventional GIC with regards to depth of cure and post-irradiation hardness (25-30).

Considering the depth of cure, VLC RMGIC should be placed in layers which by manufactures recommendations should not exceed $2 \mathrm{~mm}$. Contrary, auto-cured RMGIC (AC RMGIC) may be placed in bulk and are clinically more convenient.

The aim of this study was to assess the $\mathrm{MH}$ of $\mathrm{AC}$ RMGICs, as well as VLC RMGICs and giomer light-cured with three different curing modes of LED unit after artificial aging for up to 14 days.

Research hypotheses tested were:

1) $\mathrm{MH}$ values will be affected by material type; giomer is expected to show higher MH than GICs, whereas AC and VLC RMGICs are expected to show similar MH values

2) MH values of VLC RMGICs will be influenced by three different curing modes

3) $\mathrm{MH}$ values will improve with artificial aging.

\section{Material and methods}

\section{Microhardness measurements}

This study evaluated two VLC RMGICs: (Fuji II LC (F2LC), Photac Fil Quick Aplicap (PFQ)), two AC RMGICs: (Fuji Plus (FP), Fuji VIII GP (FVIII)) and a giomer: (Beautifil II (B2)). All tested materials were of A3 shade and their detailed composition is given in Table 1.

For each experimental group, five cylindrical specimens ( $\mathrm{d}=4 \mathrm{~mm}, \mathrm{~h}=2 \mathrm{~mm}$ ) were prepared using Teflon molds. For encapsulated materials F2LC, PFQ, FP and FVIII, the capsules were mixed according to respective manufacturers' instructions and then extruded into molds. B2 was applied into molds using a spatula, taking care not to incorporate air inclusions. Mold openings were covered with a polyethyl-
Mnogobrojne su studije uspoređivale učinkovitost halogenih i LED polimerizacijskih uređaja sa sličnom $(600 \mathrm{~mW} /$ $\mathrm{cm}^{2}$ ) (21) ili različitom jačinom svjetlosti (halogeni $350 \mathrm{mW/}$ $\mathrm{cm}^{2}$, LED $1400 \mathrm{~mW} / \mathrm{cm}^{2}$, LED $1100 \mathrm{~mW} / \mathrm{cm}^{2}$ ) (22) te $\mathrm{s} \mathrm{ra-}$ zličitom jačinom svjetlosti i vremenom izloženosti (halogen $700 \mathrm{~mW} / \mathrm{cm}^{2}$, LED $500 \mathrm{~mW} / \mathrm{cm}^{2}$; 20 s i $40 \mathrm{~s}$ ) na mikrotvrdoću (MT) SMSIC-a (23). Vrijednost mikrotvrdoće (MT-a) ovisila je o svjetlu koje je korišsteno za polimerizaciju $(21,22)$. U odnosu prema trajanju polimerizacije, slični rezultati MTa dobiveni su halogenim i LED polimerizacijskim uređajima uz izloženost od 40 sekunda, a znatne su razlike zabilježene između izloženosti halogenim uređajima u trajanju od 40 sekunda i LED-u u trajanju od 20 sekunda (23). Parisay i suradnici (24) također su uspoređivali halogene polimerizacijske uređaje i LED uređaje uz različitu jačinu svjetla (halogen $600 \mathrm{~mW} / \mathrm{cm}^{2}$, LED $700 \mathrm{~mW} / \mathrm{cm}^{2}$ ) i vrijeme polimerizacije (20 s, 30 s, 40 s) na MT gornje i donje površine uzorka. MT na gornjoj površini bio je viši u svim eksperimentalnim skupinama. Uz produljeno vrijeme polimerizacije, zabilježeno je povećanje MT-a na gornjoj i donjoj površini uzorka. U halogenoj skupini nije bilo značajnih razlika između 20, 30 i 40 sekunda, a u LED skupini 40-sekundna polimerizacija pokazala je znatno bolje rezultate od 20 - i 30-sekundne.

I u drugim istraživanjima uspoređivan je MT smolom modificiranih i konvencionalnih SIC-ova u odnosu prema dubini polimerizacije i tvrdoći nakon osvjetljivanja $(25-30)$.

$S$ obzirom na dubinu polimerizacije, SP SMSIC-ovi trebali bi se postavljati u slojevima koji prema preporukama proizvođača ne bi smjeli prelaziti 2 milimetra. Nasuprot tomu, samostvrdnjavajući SMSIC-ovi (SS SMSIC-ovi) mogu biti postavljeni u debelom sloju i klinički su prikladniji.

Cilj ovog istraživanja bio je ispitati mikrotvrdoću SS SMSIC-a te SP SMSIC-a i giomera polimeriziranih trima različitim polimerizacijskim programima LED uređaja tijekom umjetnog starenja od 14 dana.

Ispitivale su se sljedeće hipoteze:

1) vrsta materijala utjecat će na vrijednosti MT-a, očekuje se da će giomer imati veći MT od SIC-ova, a SS i SP SMSIC-ovi trebali bi imati slične vrijednosti MT-a,

2) polimerizacijski programi utjecat će na MT SP SMSICova,

3) MT materijala rast će s duljinom umjetnog starenja.

\section{Metoda}

\section{Mjerenje mikrotvrdoće}

U ovom radu ispitana su dva SP SMSIC-a (Fuji II LC (F2LC), Photac Fil Quick Aplicap (PFQ)), dva SS SMSIC-a (Fuji Plus (FP), Fuji VIII GP (FVIII)) i jedan giomer (Beautifil II (B2)). Svi testirani materijali bili su u nijansi A3, a njihov detaljni sastav naveden je u tablici 1.

U svakoj eksperimentalnoj skupini pripremljeno je pet cilindričnih uzoraka $(\stackrel{s}{s}=4 \mathrm{~mm}, \mathrm{v}=2 \mathrm{~mm}) \mathrm{s}$ pomoću teflonskih kalupa. Za kapsulirane materijale F2LC, PFQ, FP i FVIII kapsule su miješane u skladu s preporukama proizvođača, a zatim je materijal iz kapsule istisnut u kalupe. B2 je u kalupe stavljen instrumentom 5/6 prema Heidemannu, pazeći pritom da se ne unese zrak. Otvori kalupa pokriveni su 
Table 1 Materials used for the measurement of Vickers microhardness

Tablica 1. Materijali upotrijebljeni za mjerenje mikrotvrdoće prema Vickersu

\begin{tabular}{|c|c|c|c|c|c|}
\hline $\begin{array}{c}\text { Kod materijala } \\
\text { Material code }\end{array}$ & $\begin{array}{l}\text { Ime proizvoda } \\
\text { Product name }\end{array}$ & $\begin{array}{l}\text { Vrsta materijala } \\
\text { Type of material }\end{array}$ & $\begin{array}{l}\text { Proizvođač } \\
\text { Manufacturer }\end{array}$ & $\begin{array}{c}\text { Nijansa } \bullet \text { Shade } \\
\text { EXP } \\
\text { LOT }\end{array}$ & Sastav $\bullet$ Composition \\
\hline F2LC & Fuji II LC & $\begin{array}{l}\text { Smolom modificirani GIC } \\
\text { Svjetlom stvrdnjavajući } \\
\text { Restauracijski • Resin- } \\
\text { modified GIC, } \\
\text { Visible light-cured, } \\
\text { Restorative }\end{array}$ & $\begin{array}{l}\text { GC Corporation, } \\
\text { Tokio, Japan • } \\
\text { Tokyo, Japan }\end{array}$ & $\begin{array}{l}\text { A3 } \\
2018-10-26 \\
1610278\end{array}$ & $\begin{array}{l}\text { Tekućina: poliakrilna kiselina; HEMA;2,2,4 } \\
\text { TMHEDC; TEGDMA. } \\
\text { Prah: fluoroalumosilikatno staklo • Liquid: } \\
\text { polyacrylic acid; HEMA;2,2,4 TMHEDC; } \\
\text { TEGDMA. } \\
\text { Powder: fluoro-alumino-silicate glass. }\end{array}$ \\
\hline PFQ & $\begin{array}{l}\text { Photac Fil } \\
\text { Quick Aplicap }\end{array}$ & $\begin{array}{l}\text { Smolom modificirani GIC } \\
\text { Svjetlom stvrdnjavajući } \\
\text { Restauracijski • Resin- } \\
\text { modified GIC, } \\
\text { Visible light-cured, } \\
\text { Restorative }\end{array}$ & $\begin{array}{l}\text { 3M, Seefeld, } \\
\text { Njemačka } \bullet \\
\text { Germany }\end{array}$ & $\begin{array}{l}\text { A3 } \\
2019-02 \\
669852\end{array}$ & $\begin{array}{l}\text { Tekućina: staklenoionomerni kompatibilni } \\
\text { monomeri i oligomeri; akrilno- i maleinsko- } \\
\text { kiselinski kopolimeri; kamforkinon; } \\
\text { stabilizatori; voda } \\
\text { Prah: Na-Ca-Al-La fluorsilikatno staklo; } \\
\text { aminoaktivator • Liquid: glass ionomer } \\
\text { compatible monomers and oligomers; } \\
\text { acrylic- and maleic-acid copolymers; } \\
\text { camphorquinone; stabilizers; water. } \\
\text { Powder: Na-Ca-Al-La fluorosilicate glass; } \\
\text { amine activator. }\end{array}$ \\
\hline FVIII & Fuji VIII GP & $\begin{array}{l}\text { Smolom modificirani GIC } \\
\text { Samostvrdnjavajući } \\
\text { Restauracijski } \bullet \text { Resin- } \\
\text { modified GIC, } \\
\text { Auto-cured, } \\
\text { Restorative }\end{array}$ & $\begin{array}{l}\text { GC Corporation, } \\
\text { Tokio, Japan • } \\
\text { Tokyo, Japan }\end{array}$ & $\begin{array}{l}\text { A3 } \\
2019-08-06 \\
1708071\end{array}$ & $\begin{array}{l}\text { Tekućina: 2-HEMA } 25 \text { - } 50 \% \text {; vinska } \\
\text { kiselina 5 - } 10 \% \text {; } \\
\text { 7,7,9 (ili 7,9,9)-trimetil-4,13-diokso-3,14- } \\
\text { dioksa-5,12-diazoheksadekan-1,16-diyl } \\
\text { bismetakrilat } 1 \text { - 5\%; } \\
\text { 2-hidroksi-1,3 dimetakriloksipropan } 1 \text { - } 5 \% \text {. } \\
\text { Prah: N/A • Liquid: 2-HEMA 25-50 \%; } \\
\text { tartaric acid 5-10 \%; } \\
\text { 7,7,9 (or 7,9,9)-trimethyl-4,13-dioxo- } \\
\text { 3,14-dioxa-5,12-diazahexadecane-1,16-diyl } \\
\text { bismethacrylate 1-5 \%; } \\
\text { 2-Hydroxy-1,3 dimethacryloxypropane 1-5 \% } \\
\text { Powder: N/A }\end{array}$ \\
\hline FP & Fuji PLUS & $\begin{array}{l}\text { Smolom modificirani GIC } \\
\text { Samostvrdnjavajući } \\
\text { Cementiranje } \bullet \text { Resin- } \\
\text { modified GIC } \\
\text { Auto-cured, } \\
\text { Luting }\end{array}$ & $\begin{array}{l}\text { GC Corporation, } \\
\text { Tokio, Japan • } \\
\text { Tokyo, Japan }\end{array}$ & $\begin{array}{l}\text { A3 } \\
2019-07-19 \\
1707201\end{array}$ & $\begin{array}{l}\text { Tekućina: 2-HEMA } 25-50 \% \text {; } \\
\text { 7,7,9 (ili 7,9,9)-trimetil-4,13-diokso-3,14- } \\
\text { dioksa-5,12- diazoheksadekan -1,16-diyl } \\
\text { bismetakrilat } 1 \text { - 5\%; } \\
\text { 2-hidroksi-1,3 dimetakriloksipropan } 1 \text { - } 5 \% \text {. } \\
\text { Prah: N/A • Liquid: 2-HEMA 25-50 \%; } \\
\text { 7,7,9 (or 7,9,9)-trimethyl-4,13-dioxo- } \\
\text { 3,14-dioxa-5,12-diazahexadecane-1,16-diyl } \\
\text { bismethacrylate 1-5\%; } \\
\text { 2-Hydroxy-1,3 dimethacryloxypropane 1-5\% } \\
\text { Powder: N/A }\end{array}$ \\
\hline B2 & Beautifil II & $\begin{array}{l}\text { Giomer, } \\
\text { Svjetlom stvrdnjavajući, } \\
\text { Restauracijski • Giomer, } \\
\text { Visible light-cured, } \\
\text { Restorative }\end{array}$ & $\begin{array}{l}\text { Shofu Inc., Kjoto, } \\
\text { Japan }\end{array}$ & $\begin{array}{l}\text { A3 } \\
2020-04-30 \\
051705\end{array}$ & $\begin{array}{l}\text { Bis-GMA 7.5\%, trietilen-glikol dimetakrilat } \\
5 \% \text {, alumofluoroborosilikatno staklo } 7.5 \\
\%, \mathrm{Al}_{2} \mathrm{O}_{3}, \text { DL-kamforkinon. } \bullet \text { Bis-GMA } \\
7.5 \% \text {, Triethylenglycol dimethacrylate } 5 \% \text {, } \\
\text { Aluminofluoro-borosilicate glass } 7.5 \%, \mathrm{Al}_{2} \mathrm{O}_{3} \text {, } \\
\text { DL-Camphorquinone. }\end{array}$ \\
\hline
\end{tabular}

$(2,2,4$ TMHEDC $=2,2,4$ trimetil heksametilen dikarbonat; HEMA = 2-hidroksietilmetakrilat; TEGDMA = trietilen glikol dimetakrilat $) \bullet(2,2,4$ TMHEDC = 2,2,4 trimethyl hexamethylene dicarbonate; HEMA = 2-hydroxyethylmethacrylate; TEGDMA = triethylene glycol dimethacrylate)

ene terephthalate (PET) film and compressed using a stainless steel slab to remove the excess material. For the light-curable materials, specimens were illuminated with a LED LCU (Bluephase G2, Ivoclar-Vivadent, Schaan, Liechtenstein) positioned immediately above the specimen. Each experimental group was illuminated with one of the following light-curing modes for $20 \mathrm{~s}:$ low $\left(650 \mathrm{~mW} / \mathrm{cm}^{2}\right)$, soft $(650-1100 \mathrm{~mW} /$ $\left.\mathrm{cm}^{2}\right)$ and high $\left(1100 \mathrm{~mW} / \mathrm{cm}^{2}\right)$. Specimen preparation was performed at room temperature, while subsequent aging was simulated by the storage at $37 \pm 1^{\circ} \mathrm{C}$ in deionized water in an filmom od polietilen-tereftalata (PET) i stisnuti pločom od nehrđajućeg čelika kako bi se uklonio višak materijala. Za materijale koji se polimeriziraju svjetlom, uzorci su polimerizirani LED polimerizacijskim uređajem (Bluephase G2, Ivoclar-Vivadent, Schaan, Lihtenštajn) koji je postavljen izravno iznad uzorka. Svaka eksperimentalna skupina osvijetljena je jednim od sljedećih programa polimerizacije tijekom 20 sekunda: niskim $\left(650 \mathrm{~mW} / \mathrm{cm}^{2}\right)$, postupnim $(650-1100 \mathrm{~mW}$ $\left.\mathrm{cm}^{2}\right)$ i visokim $\left(1100 \mathrm{~mW} / \mathrm{cm}^{2}\right)$. Priprema uzoraka obavljena je na sobnoj temperaturi, a umjetno starenje poslije toga 
incubator (Cultura, Ivoclar-Vivadent, Schaan, Liechtenstein) for up to 14 days.

Vickers $\mathrm{MH}$ was evaluated at four time intervals: immediately after specimen preparation and manufacturer recommended setting time 0 day, 1 day, 7 days and 14 days. The surfaces of aged specimens were gently blotted to remove water before the measurements. A diamond pyramid was indented into irradiated specimen surfaces using the Leitz Miniload 2 microhardness tester (Leitz, Oberkochen, Germany) with a load of $100 \mathrm{~g}$, dwell time of $10 \mathrm{~s}$ and 5 indentations per specimen. $\mathrm{MH}$ was calculated by the following equation: $\mathrm{HV}=1.8544 \mathrm{xF} / \mathrm{d}^{2}$, where $\mathrm{d}$ is the diagonal of the indentation and $\mathrm{F}=\mathrm{m} \times \mathrm{g}(\mathrm{g}=9.81 \mathrm{~N} / \mathrm{kg}, \mathrm{m}=\mathrm{load})$.

\section{Statistical analysis}

Normality of distribution and homogeneity of variances was confirmed using the Shapiro-Wilk's and the increase of both the top and the bottom MH was observed the Levene's test, respectively. The assumption of sphericity for repeated measurements was confirmed using the Mauchly's test. Mean values of $\mathrm{MH}$ for each experimental group were compared using a mixed model ANOVA, which accounted for the clustering effect of the repeated measurements performed on individual specimens (31). Post-hoc multiple comparisons were made using a Tukey's HSD correction. Partial eta-squared statistics were used to evaluate relative influences of the factors "time", "material" and "curing mode". Statistical software SPSS 20 (IBM, Armonk, NY, USA) was used, with the level of significance set at 0.05 .

\section{Results}

Mean $\mathrm{MH}$ values as a function of material, aging time and curing mode are presented in Figure 1. The full factorial ANOVA which considered the light-cured materials B2, F2LC and PFQ identified a highly significant $(p<0.001)$ effect of the factors "material", "time" and "curing mode", with the relative influences of individual factors represented by partial eta squared values of $0.991,0.778$ and 0.171 , respectively.

The highest partial eta squared for the factor "material" indicates that the highest amount of variation of the dependent variable $\mathrm{MH}$ is attributable to the material type. For individual materials, $\mathrm{MH}$ values were in the following ranges: 70.4-74.4 for B2, 34.1-44.1 for F2LC, 26.7-41.1 for PFQ, 31.0-40.5 for FP and 27.8-38.6 for and FVIII.

The factor "time" showed a significant interaction $(p<0.001)$ with the factor "material", reflecting a more pronounced $\mathrm{MH}$ improvement with time in the group of glassionomer-based materials compared to the giomer B2. After 14 days, the $\mathrm{MH}$ values for $\mathrm{B} 2$ improved for 2.9-5.7\% compared to the immediate (0-day) values, whereas a much more extensive improvement was identified in the glassionomer-based materials: $20.9-22.4 \%$ in F2LC, $47.7-51.0 \%$ in PFQ, 30.9\% in FP and 38.7\% in FVIII.

Although statistically significant, the influence of the factor "curing mode" on $\mathrm{MH}$ was the weakest among all tested factors. This modest effect of different curing modes is visu- simulirano je pohranom u deioniziranoj vodi $\mathrm{u}$ inkubatoru (Cultura, Ivoclar-Vivadent, Schaan, Lihtenštajn) pri $37 \pm 1$ ${ }^{\circ} \mathrm{C}$ u razdoblju do 14 dana.

MT prema Vickersu mjeren je u četiri vremenska intervala: 0 . dan, tj. neposredno nakon pripreme uzorka i nakon vremena stvrdnjivanja prema preporuci proizvođača, 1 . dan, 7. dan i 14. dan. Prije mjerenja s površine pohranjenih uzoraka nježno je uklonjena voda. Dijamantna piramida utisnuta je u stvrdnutu površinu uzoraka primjenom uređaja za mjerenje mikrotvrdoće Leitz Miniload 2 (Leitz, Oberkochen, Njemačka) opterećenjem od 100 g, trajanjem opterećenja 10 sekunda i s 5 utisnuća po uzorku. MT je izračunat prema sljedećoj jednadžbi: $\mathrm{MT}=1,8544 \times \mathrm{F} / \mathrm{d}^{2}$, pri čemu je d dijagonala utisnuća i $\mathbf{F}=\mathrm{m} \times \mathrm{g}(\mathrm{g}=9,81 \mathrm{~N} / \mathrm{kg}, \mathrm{m}=$ teret $)$.

\section{Statistička analiza}

Normalnost distribucije i homogenost varijanci potvrđena je najprije Shapiro-Wilkovim testom, a zatim Leveneovim. Pretpostavka sferičnosti za ponovljena mjerenja potvrđena je Mauchlyjevim testom. Srednje vrijednosti MT-a za svaku eksperimentalnu skupinu uspoređene su miješanim modelom ANOVA-e kojim je uzet u obzir učinak grupiranja ponovljenih mjerenja obavljenih na pojedinačnim uzorcima (31). Mnoge post-hoc usporedbe obavljene su Tukeyjevim HSD testom. Statistika parcijalnog eta-kvadrat testiranja korištena je za procjenu relativnih učinaka čimbenika vrijeme, materijal i polimerizacijski program. Korišten je statistički softver SPSS 20 (IBM, Armonk, NY, SAD), uz razinu značajnosti 0,05 .

\section{Rezultati}

Srednje vrijednosti MT-a kao funkcije materijala, vremena starenja i polimerizacijskog programa prikazane su na slici 1. Potpuna faktorska ANOVA kojom su uzeti u obzir svjetlom polimerizirajući materijali B2, F2LC i PFQ zabilježila je iznimno značajan $(p<0,001)$ učinak čimbenika materijal, vrijeme i polimerizacijski program, uz razmjerne utjecaje pojedinačnih čimbenika prikazanih parcijalnim eta-kvadrat vrijednostima od 0,991, 0,778 i 0,171 (tim redom).

Najviša vrijednost parcijalnog eta-kvadrata za čimbenik materijal pokazuje da se najveće varijacije ovisne varijable MT-a mogu pripisati vrsti materijala. Za pojedinačni materijal vrijednosti MT-a bile su se u sljedećim intervalima: 70,4 - 74,4 za B2, 34,1 - 44,1 za F2LC, 26,7 - 41,1 za PFQ, 31,0 - 40,5 za FP i 27,8 - 38,6 za FVIII.

Čimbenik vrijeme pokazao je značajnu interakciju $(p<$ $0,001)$ s čimbenikom materijal, odražavajući izraženije poboljšanje MT-a s vremenom u skupini stakloionomernih materijala u usporedbi s giomerom B2. Nakon 14 dana vrijednosti MT-a za B2 poboljšale su se za 2,9 do 5,7 \% u usporedbi s neposrednim (0. danom) vrijednostima, a znatno veće poboljšanje zabilježeno je za stakloionomerne materijale: $20,9-22,4 \%$ u F2LC-u, 47,7 - 51,0 \% u PFQ-u, 30,9 \% u FP-u i 38,7 \% u FVIII.

Iako je statistički značajan, učinak čimbenika polimerizacijski program na MT bio je najslabiji od svih ispitanih čimbenika. Taj skromni učinak različitih polimerizacijskih 


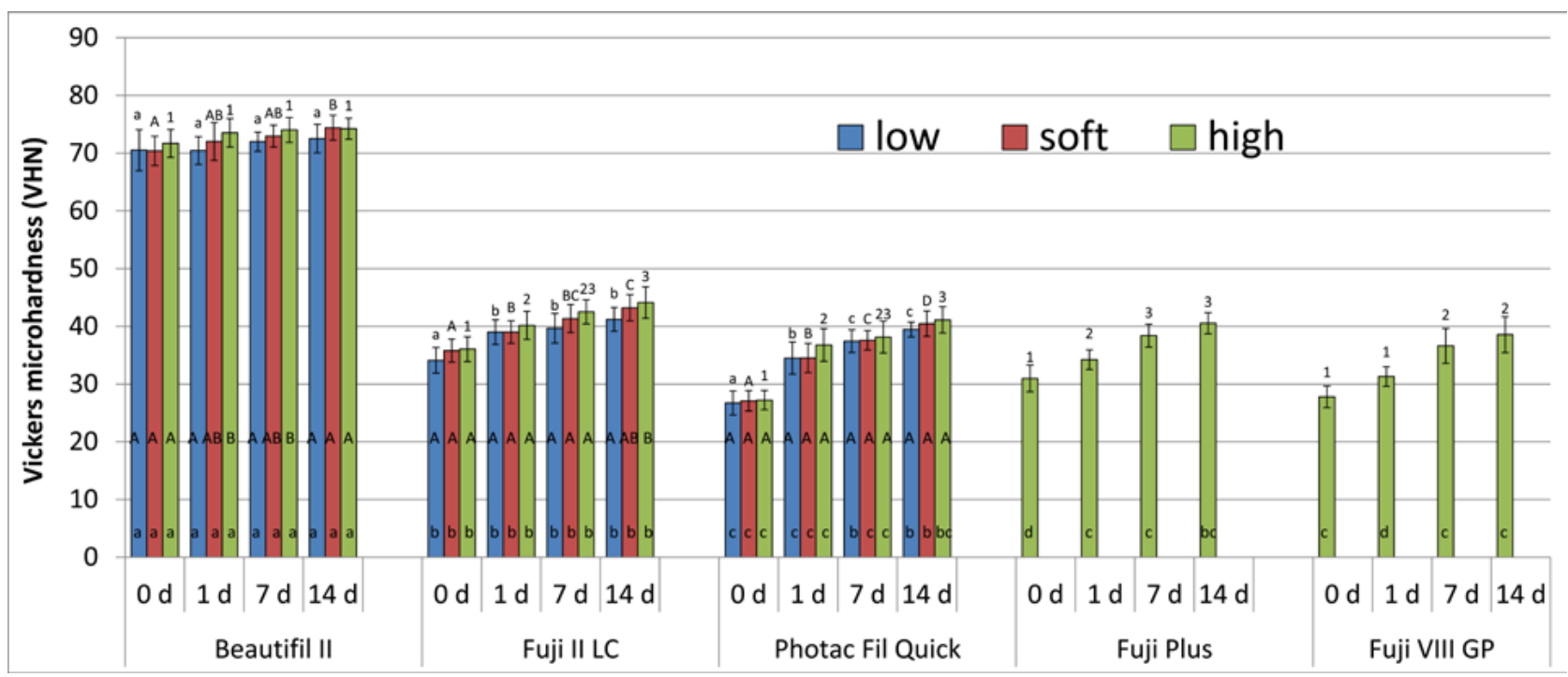

Figure 1 Mean values ( \pm s. d.) of microhardness (in Vickers hardness numbers) for tested materials as a function of curing mode and postirradiation time intervals. Same letters and numbers above the bars denote statistically homogeneous groups among time points, within each curing mode as follows: lowercase letters - "low", uppercase letters - "soft" and numbers - "high". Same uppercase letters at the middle of the bars represent statistically homogeneous groups for the comparison among curing modes. Same lowercase letters at the bottom of the bars denote statistically homogeneous groups for the comparison among materials.

Slika 1. Srednje vrijednosti $( \pm$ s. d.) mikrotvrdoće (u brojevima tvrdoće prema Vickersu) za testirane materijale kao funkcija programa stvrdnjivanja i u vremenskim intervalima nakon zračenja. Ista slova i brojke iznad stupaca označuju statistički homogene skupine među vremenskim točkama i unutar svakoga programa stvrdnjivanja kako slijedi: mala tiskana slova - niski, velika tiskana slova - postupni i brojke - visoki; ista velika tiskana slova u sredini stupaca označuju statistički homogene skupine za usporedbu među programima stvrdnjivanja; ista mala tiskana slova na dnu stupaca predstavljaju statistički homogene skupine za usporedbu među materijalima

ally observable in Figure 1 within all combinations of factors "material" and "time" as a trend of increase in the order of low < soft < high. However, due to the small effect size, the differences among curing modes were statistically significant only in B2 after 1 and 7 days and in F2LC after 14 days. In these cases, the $\mathrm{MH}$ appeared significantly higher for the "high" curing mode compared to the "low" mode.

\section{Discussion}

The aim of this study was to determine the $\mathrm{MH}$ of $\mathrm{AC}$ and VLC RMGICs, as well as a giomer and to determine a dependence of the curing type (AC/VLC) and storage time on the $\mathrm{MH}$ as well as a dependence of three different curing modes ("low", "soft", "high") of the LED curing unit and storage time.

In previous studies, the dependence of the $\mathrm{MH}$ of composite materials on the applied polymerization modes of halogen curing units was demonstrated. In a study by Soh and Yap (32) the polymer structure of composite materials was curing mode dependent. Pulse delay curing mode resulted in lower crosslink density and lower Knoop hardness. In da Silva's research (33), higher values of Knoop MH of composite materials were found by exposing the material to high polymerization mode instead of standard and gradual.

A high power LED LCU was chosen in this study since such a curing unit type is the most frequently used in current clinical practice. Hardness was evaluated after four postirradiation times, starting immediately after manufacturer recommended curing time and followed by artificial aging for 1,7 and 14 days. For the first measurement, the sam- programa može se vidjeti na slici 1 . kod svih kombinacija čimbenika materijal i vrijeme kao trend povećanja prema redoslijedu niski< postupni < visoki. No zbog malog učinka, razlike između polimerizacijskih programa statistički su značajne samo za B2 nakon jednog dana i 7 dana te za F2LC nakon 14 dana. U tim je slučajevima MT znatno viši za viso$k i$ polimerizacijski program u usporedbi s niskim.

\section{Rasprava}

Cilj ovog istraživanja bio je odrediti mikrotvrdoću SS SMSIC-ova i SP SMSIC-ova i giomera te utvrditi ovisnost MT-a o načinu stvrdnjivanja materijala (samostvrdnjivanje/svjetlosna polimerizacija) i vremenu pohrane, te ovisnost MT-a o trima različitim polimerizacijskim programima (niski, postupni, visoki) LED polimerizacijskog uređaja i vremenu pohrane.

U prijašnjim istraživanjima istaknuta je ovisnost MT-a kompozitnih materijala o primijenjenom polimerizacijskom programu halogenih polimerizacijskih uređaja. U studiji Soha i Yapa (32) polimerna struktura kompozitnog materijala bila je ovisna o polimerizacijskom programu. Program polimerizacije Pulse delay rezultirao je slabijom gustoćom polimerne mreže i slabijom tvrdoćom prema Knoopu. U da Silvinu istraživanju (33) više vrijednosti MT-a kompozitnih materijala prema Knoopu zabilježene su nakon izlaganja materijala visokom programu polimerizacije umjesto standardnom i postupnom.

U ovom radu odabran je LED polimerizacijski uređaj jake snage zato što se takav uređaj najčešće koristi u suvremenoj kliničkoj praksi. Tvrdoća je određena četiri puta nakon 
ples were without contact to storage media, thus simulating a clinical situation in which a restoration is isolated from saliva and water during the placement and light-curing. Afterwards, the restorations were continuously exposed to wet environment throughout their whole service life. Although it is known that $\mathrm{MH}$ varies throughout the recommended layer thickness $(23,31)$, the present study focused on the effect of different light-curing modes on $\mathrm{MH}$ measured on the specimen surface, in accordance to previous studies (27).

Among all tested materials, B2 showed the highest values of $\mathrm{MH}$ which were nearly two times higher than these of the RMGICs tested. Such a finding was expected because of the giomer composition close to composite resins, despite the difference in inorganic filler.

The $\mathrm{MH}$ values of $\mathrm{B} 2$ generally ranged from $70.4 \pm 2.5$ to $74.4 \pm 2.2$ (Figure 1). These findings are comparable to the results reported by Mobarak et al. (22) in a study on the influence of different LCU (halogen $350 \mathrm{~mW} / \mathrm{cm}^{2}$, LED 1400 $\mathrm{mW} / \mathrm{cm}^{2}$, LED $1100 \mathrm{~mW} / \mathrm{cm}^{2}$ ). The highest $\mathrm{MH}$ values of Beautifil, which is compositionally similar to B2, measured fifteen minutes after curing were achieved by Bluephase LCU (LED $1100 \mathrm{~mW} / \mathrm{cm}^{2}$ ), which agrees with our $\mathrm{MH}$ values obtained in high curing mode immediately after curing (72.9 $\pm 0.9,71.7 \pm 2.4$ respectively).

During the 14 days of artificial aging, $\mathrm{MH}$ values for $\mathrm{B} 2$ showed a small but continuous increase. Yap et al. (34) evaluated the hardness of Beautifil, after the 30 days storage in distilled water and reported $\mathrm{MH}$ of $72.7 \pm 3.8$, which is comparable to our results after 14 days, especially to those obtained in low mode because they used LCU with the mean intensity of $460 \pm 4 \mathrm{~mW} / \mathrm{cm}^{2}$. Unfortunately, the cited study did not evaluate $\mathrm{MH}$ at earlier time points, thus providing no data for comparison with our results.

VLC RMGICs exhibited a similar influence of curing modes on $\mathrm{MH}$ as it was in $\mathrm{B} 2$.

A statistically significant effect of the curing mode identified in the omnibus test suggests that $\mathrm{MH}$ was positively related to the total radiant energy received by specimen surfaces. Radiant energy (J) delivered by each curing mode (low 9.9, soft 14.7, high 17.5) concurs with the ranking of $\mathrm{MH}$ values: low $<$ soft $<$ high (35). The inability of the statistical analysis to detect significant differences within each material $\mathrm{x}$ time combination was due to a very small effect size. When the curing mode "high" was used instead of "low", the differences in $\mathrm{MH}$ amounted to about 1-3, which represents an improvement in the range of $2-7 \%$ ?

A similar influence of the curing modes in the polymerization process of two different material groups tested is an interesting finding since the setting mechanism of the RMGICs involves acid-base reaction and polymerization of resinous part of the cement. Both reactions are diffusion-controlled; therefore their rates decrease as the network formation impairs the diffusion of reactive species. Since the freeradical-mediated polymerization reaction is inherently faster than the acid-base reaction, the light activation causes the resinous network formation to occur ahead of the acid-base reaction, thus inhibiting it $(11,36)$. In a Fourier-transform infrared (FTIR) study of visible light-cured RMGICs Fuji osvjetljivanja - prvi put neposredno nakon proizvođačeva preporučenog vremena stvrdnjivanja i zatim nakon umjetnog starenja od 1, 7 i 14 dana. Za prvo mjerenje uzorci nisu bili u doticaju s medijem za pohranjivanje, tako se oponašalo kliničku situaciju u kojoj je restauracija izolirana od sline i vode tijekom postavljanja i svjetlosne polimerizacije. Nakon toga restauracije su trajno izložene vlažnim uvjetima tijekom njihova trajanja u ustima. Iako je poznato da MT varira kroz preporučenu debljinu sloja $(23,31)$, ovo istraživanje usredotočeno je na učinak različitih polimerizacijskih programa na MT površine uzorka, u skladu s ranijim istraživanjima (27).

Od svih ispitanih materijala, B2 je imao najveće vrijednosti MT-a koje su bile gotovo dva puta veće od vrijednosti ispitanih SMSIC-ova. Takav nalaz očekivan je zbog sličnosti sastava giomera kompozitnim smolama, unatoč razlici u anorganskom punilu.

MT-i B2 bili su u rasponu od 70,4 $\pm 2,5$ do $74,4 \pm 2,2$, slika 1 . Ti nalazi mogu se usporediti s nalazima Mobaraka i sur. (22) u studiji o utjecaju različitih polimerizacijskih uređaja (halogenih $350 \mathrm{~mW} / \mathrm{cm}^{2}$, LED $1400 \mathrm{~mW} / \mathrm{cm}^{2}$, LED $\left.1100 \mathrm{~mW} / \mathrm{cm}^{2}\right)$. Najveće vrijednosti mikrotvrdoće Beautifila $(72,9 \pm 0,9)$ čiji je sastav sličan sastavu B2, izmjerene petnaest minuta nakon stvrdnjivanja, postignute su uređajem Bluephase (LED $1100 \mathrm{~mW} / \mathrm{cm}^{2}$ ), što odgovara našim vrijednostima MT-a dobivenima tijekom polimerizacije visokim programom neposredno nakon stvrdnjivanja $(71,7 \pm 2,4)$.

Tijekom 14 dana umjetnog starenja, vrijednosti MT-a za B2 polako su, ali kontinuirano rasle. Yap i suradnici (34) ispitali su tvrdoću Beautifila nakon 30 dana pohrane u destiliranoj vodi i zabilježili su MT od 72,7 $\pm 3,8$, što se može usporediti $s$ našim rezultatima nakon 14 dana, posebno onima koji su dobiveni niskim polimerizacijskim programom jer su se koristili polimerizacijskim uređajem srednjeg intenziteta od $460 \pm 4 \mathrm{~mW} / \mathrm{cm}^{2}$. Nažalost, u citiranoj studiji nije ispi$\tan$ MT u ranijim vremenskim intervalima, zbog čega ne postoje podatci koji bi se mogli usporediti s našim rezultatima.

SP SMSIC-ovi pokazali su sličan utjecaj polimerizacijskih programa na MT kao i B2.

Statistički značajan utjecaj polimerizacijskog programa koji je dokazan omnibus-testom upućuje da je MT u pozitivnom odnosu s ukupnom energijom zračenja koju je primila površina uzoraka. Energija zračenja $(\mathrm{J})$ rabljena pri svakom programu stvrdnjivanja (nizak - 9,9 J, postupan - 14,7 J, visok - 17,5 J) odgovara rasponu vrijednosti MT-a: niska $<$ postupna $<v i-$ soka (35). Nemogućnost statističke analize da otkrije značajne razlike za svaki materijal u kombinaciji s vremenom, pojavila se zbog vrlo malog utjecaja. Kada je korišsten visoki program stvrdnjivanja umjesto niskoga, razlike u MT-u iznosile su se otprilike od 1 do 3 , što čini poboljšanje u rasponu od 2 do $7 \%$.

Sličan utjecaj polimerizacijskog programa na proces polimerizacije dviju ispitanih različitih skupina materijala zanimljiv je nalaz, zato što mehanizam stvrdnjivanja SMSIC-ova uključuje kiselo-lužnatu reakciju i polimerizaciju smolastoga dijela cementa. Obje reakcije kontrolirane su difuzijom i zato se njihov stupanj smanjuje jer formiranje mreže onemogućuje difuziju reaktivnih tvari. S obzirom na to da je reakcija polimerizacije slobodnim radikalima sama po sebi brža od kiselo-lužnate reakcije, svjetlosna aktivacija uzrokuje oblikovanje 
II LC and Photac Fil Quick Aplicap, the rate of the acidbase reaction was the highest during the light activation (37). These results were explained by the decline in the reaction rate of the diffusion-controlled acid-base reaction through the formation of an infinite methacrylate network (gelation) and additionally by the contribution of the heating effect which originated from the exothermic polymerization reaction, as well as from the curing unit (37).

However, our results showed that MH of VLC RMGICs continued to increase for 14 days post-irradiation. These findings correspond to Ellakuria et al. (26). They found increasing $\mathrm{MH}$ up to 15 days, after which $\mathrm{MH}$ decreased up to 12 months.

Young (38) investigated conventional GIC (Fuji IX) and RMGIC (Fuji II LC Improved) by FTIR investigation and observed two separate diffusion mechanisms for acid neutralization. The earlier-occurring, faster reaction stopped after 30 and 150 min respectively, suggesting that the water required for its progress was depleted after a certain time period. The slower reaction was also an acid-base neutralization which was triggered by absorbed water, therefore occurring with a delay. Thus, the post-irradiation increase in $\mathrm{MH}$ may be mainly addressed to the continuation of the acid-base reaction.

There are several materials related factors that may influence the mechanical properties of RMGIC such as resin monomer content, type of resin monomers, particle volume and size, polymeric acids molecular weight, number and size of voids and water content. From the point of $\mathrm{MH}$, particle volume and size are of particular interest.

Mean particle size of F2LC and PFQ are $5.9 \mu \mathrm{m}$ and 5.56 $\mu \mathrm{m}$ respectively (39) and even less in F2LC Improved (38).

Xie et al. (40) evaluated mechanical properties and microstructures of GICs and found that the mechanical properties were closely related to their microstructures. The Knoop hardness (KHN) obtained in their study was significantly greater for Fuji II LC Improved than for Photac Fil, as it was in our study comparing F2LC and PFQ. Based on the SEM fractography, the author suggested that in the case of Fuji II LC Improved a better indentation resistance might be attributed to homogeneously distributed small glass particles, while on the fractured surface of Photac Fil less exposed glass particles well-integrated in the resinous matrix were identified.

$\mathrm{MH}$ values for PFQ significantly increased from $0 \mathrm{~d}$ to $1 \mathrm{~d}$, followed by a less pronounced trend of increase towards $14 \mathrm{~d}$. This evidence might be explained by research of Kakaboura et al. (41) who used FTIR analysis and found that acid-base reaction of F2LC, Photac-Fil Aplicap (earlier formulation of PFQ) and Vitremer was significantly slowed by the light polymerization. Between the tested materials, the smallest extent of the acid-base reaction was found in Photac-Fil Aplicap. The authors attributed these findings to the majority of its polyacrylic acid added to powder component, which required more water infiltration for full reaction. This could explain significantly lower $\mathrm{MH}$ values measured for $\mathrm{PFQ}$ in our study at $0 \mathrm{~d}$ in all three curing modes when samples were without contact to storage media. After 1 day aging in de- smolaste mreže prije kiselo-lužnate reakcije, čime je inhibira $(11,36)$. U istraživanju s pomoću infracrvene Fourier-Transform (FTIR) analize svjetlom stvrdnjavajućih SMSC-ova Fuji II LC i Photac Fil Quick Aplicap, brzina kiselo-lužnate reakcije bila je najveća tijekom aktivacije svjetlom (37). Ti su rezultati objašnjeni padom u procesu kiselo-lužnate reakcije kontroliranom difuzijom te stvaranjem mreže metakrilata (gelacije) i dodatno doprinosom zagrijavanja koji se pojavljuje zbog egzotermičke reakcije polimerizacije te zagrijavanja polimerizacijskog uređaja (37).

No naši rezultati pokazali su da se MT SP SMSIC-a nastavila povećavati 14 dana nakon osvjetljivanja. Ta zapažanja odgovaraju rezultatima Ellakurija i suradnika (26). Oni su povećanje MT-a zabilježili do 15 dana, nakon čega se smanjivala do 12 mjeseci.

Young (38) je istraživao konvencionalni SIC (Fuji IX) i SMSIC (Fuji II LC Improved) analizom FTIR i zabilježio dva odvojena mehanizma difuzije za neutralizaciju kiseline. Reakcija koja se pojavljivala ranije i bila je brža, prestala je nakon 30 i 150 minuta (tim slijedom), što sugerira da se voda koja je potrebna za njezin nastavak potrošila nakon određenog vremena. Sporija reakcija također je bila kiselo-lužnata neutralizacija koju je potaknula apsorbirana voda te se zato pojavila s odgodom. Zato se povećanje MT-a nakon osvjetljivanja uglavnom može pripisati nastavljanju kiselo-lužnate reakcije.

Nekoliko je čimbenika vezanih uz vrstu materijala koji mogu utjecati na mehanička svojstva SMSIC-ova, kao što su sadržaj smolastog monomera, tip smolastih monomera, veličina i opseg čestica, molekularna težina polimernih kiselina, broj i veličina pora te sadržaj vode. Za MT su opseg i veličina čestica od posebnog interesa.

Srednja veličina čestica F2LC i PFQ jest 5,9 $\mu \mathrm{m}$ i 5,56 $\mu \mathrm{m}$ (39), a čak i manja u F2LC Improvedu (38).

Xie i suradnici (40) ispitali su mehanička svojstva i mikrostrukturu SIC-a i ustanovili da su mehanička svojstva usko povezana s mikrostrukturom. Tvrdoća prema Knoopu (KHN), dobivena tijekom njihova istraživanja, znatno je veća za Fuji II LC Improved negoli za Photac Fil, kao i u našem istraživanju u kojemu su uspoređeni F2LC i PFQ. Na osnovi SEM fraktografije, autor ističe da se u slučaju Fuji II LC Improveda veći otpor na utiskivanje može pripisati homogeno distribuiranim malim česticama stakla, a na lomnoj površini Photac Fila zabilježene su manje izložene čestice stakla dobro integrirane u smolastu matricu.

Vrijednosti MT-a za PFQ značajno su se povećale od 0 . o 1. dana, nakon čega je slijedio slabiji trend povećanja prema 14. danu. Ovaj nalaz može se objasniti radom Kakaboura i suradnika (41) koji su, koristeći se analizom FTIR, ustanovili da je kiselo-lužnata reakcija F2LC-a, Photac-Fil Aplicapa (ranije formulacije PFQ-a) i Vitremera znatno usporena procesom polimerizacije. Među ispitanim materijalima najmanji stupanj kiselo-lužnate reakcije zabilježen je za PhotacFil Aplicap. Autori su takva zapažanja pripisali poliakrilnoj kiselini koja je uglavnom dodana komponenti praha, što za potpunu reakciju zahtijeva infiltraciju veće količine vode. To bi moglo objasniti znatno niže vrijednosti MT-a izmjerene za PFQ u našoj studiji na 0 . dan u svim trima polimerizacijskim programima kada uzorci nisu bili u doticaju s vo- 
ionized water, water sorption initiated more acid-base reaction resulting in significantly increased $\mathrm{MH}$. Between tested materials, including AC RMGICs, such behaviour was only observed in PFQ and this could be due to the proprietary material composition which is not fully disclosed by the manufacturer.

AC RMGICs, FP, and FVIII, although for different clinical use (luting of indirect restorations and restorative treatment, respectively) exhibited $\mathrm{MH}$ values in the range of VLC RMGICs. Also, the influence of aging time was similar to that observed in VLC RMGIC products. It can be assumed that despite the monomer polymerization in AC RMGICs is longer than in VLC RMGICs, monomer polymerization influenced the acid-base reaction in the same way in both material groups, resulting in a continuation of the and acid-base reaction in tested time period, which was reflected as an increase in $\mathrm{MH}$.

$\mathrm{MH}$ values attained by different curing modes were positively related to radiant energy delivered to the specimen surface. Despite being statistically significant, the differences among curing modes can be considered negligible from the clinical standpoint. Material composition exerted a stronger effect on $\mathrm{MH}$ values, suggesting that the material choice had more impact on the mechanical properties than the curing mode. An improvement in $\mathrm{MH}$ was identified in all materials after an artificial aging of 14 days, reflecting the long-term continuation of the setting reaction.

\section{Conclusions}

All curing modes investigated can be effectively used in clinical work. Material type had more impact on microhardness than the curing mode.

\section{Acknowledgements}

Dental companies 3M (Seefeld, Germany) and GC Corporation (Tokyo, Japan) are gratefully acknowledged for donation of materials.

\section{Conflict of interest}

None declared denim medijem u kojemu su bili pohranjeni. Nakon jednog dana starenja u deioniziranoj vodi, apsorpcija vode potaknula je kiselo-lužnatu reakciju, što je rezultiralo znatnim povećanjem MT-a. Među ispitanim materijalima, uključujući i SS SMSIC-ove, takvo je ponašanje zabilježeno samo za PFQ i moglo bi biti posljedica tvorničkog sastava materijala koji proizvođač ne otkriva u cijelosti.

SS SMSIC-ovi, FP-ovi i FVIII, iako se upotrebljavaju u različite kliničke svrhe (za cementiranje indirektnih nadomjestaka i restauracijski ispun - tim redom), imali su vrijednosti MT-a u rasponu SP SMSIC-ova. Utjecaj vremena starenja također je bio sličan kao i za SP SMSIC-ove. Moguća pretpostavka jest da, iako polimerizacija monomera kod SS SMSIC-a traje dulje negoli kod SP SMSIC-a, polimerizacija monomera utjecala je na kiselo-lužnatu reakciju na jednak način na obje skupine materijala, što je rezultiralo nastavkom kiselo-lužnate reakcije tijekom ispitivanja, a to se odrazilo kao povećanje MT-a kroz vrijeme.

Vrijednosti MT-a dobivene različitim polimerizacijskim programima u pozitivnom su odnosu s energijom osvjetljivanja koja je dospjela na površinu uzorka. Unatoč tomu što su statistički značajne, razlike polimerizacijskih programa mogu se smatrati zanemarivima s kliničkog stajališta. Sastav materijala snažnije je utjecao na vrijednosti MT-a, što pokazuje da je odabir materijala imao veći utjecaj na mehanička svojstva od polimerizacijskog programa. Poboljšanje u MT-u zabilježeno je za sve materijale nakon umjetnog starenja od 14 dana, što odražava dugotrajno nastavljanje reakcije stvrdnjivanja.

\section{Zaključak}

Svi ispitivani polimerizacijski programi mogu se učinkovito upotrebljavati u kliničkom radu. Vrsta materijala imala je veći učinak na mikrotvrdoću od samog polimerizacijskog programa.

\section{Zahvale}

Srdačno zahvaljujemo dentalnim tvrtkama 3M (Seefeld, Njemačka) i GC Corporation (Tokio, Japan) na donaciji materijala.

\section{Sukob interesa}

Nije bilo sukoba interesa.

\section{Sažetak}

Svrha: U ovom radu procijenjen je učinak polimerizacijskih programa na površinsku mikrotvrdoću svjetlom polimerizirajućih staklenoionomernih cemenata (SP SMSIC) modificiranih smolom i giomera nakon različitih vremena pohrane u usporedbi sa samostvrdnjujućim staklenoionomernim cementima (SS SMSIC) modificiranima smolom. Materijali i metode: Korišteni su sljedeći materijali: SP SMSIC: Fuji II LC, Photac Fil Quick Aplicap, SS SMSIC: Fuji Plus, Fuji VIII i Giomer: Beautifil II. Mjerenja mikrotvrdoće obavljena su metodom prema Vickersu (100 g mase tijekom $10 \mathrm{~s}$ ) u sljedećim vremenskim intervalima: neposredno nakon preporučenog stvrdnjavanja i poslije $1,7 \mathrm{i} 14$ dana pohranjenosti u destiliranoj vodi. Pet uzoraka $(\mathrm{d}=4 \mathrm{~mm}, \mathrm{~h}=2 \mathrm{~mm}$ ) pripremljeno je za svaki program stvrdnjavanja i ispitivani materijal. Rezultati: Nakon 14 dana poboljšanje mikrotvrdoće dokazano je u svim ispitanim materijalima. Primjenom potpunoga faktorskog testa ANOVA-e utvrđena je visoka značajnost $(\mathrm{p}<0,001)$ utjecaja čimbenika materijal, vrijeme i polimerizacijski program (niski, postupni, visoki) za svjetlom polimerizirajuće materijale Beautifil II, Fuji II LC i Photac Fil Quick. Uočena je i statistički značajna razlika u mikrotvrdoći između različitih materijala (Beautifil II > Fuji II LC > Photac Fil Quick > Fuji Plus > Fuji VIII) i programa stvrdnjivanja (niski < postupni < visoki). Zaključci: Vrsta materijala najviše je utjecala na mikrotvrdoću, slijedi čimbenik vrijeme, a polimerizacijski programi pokazali su znatno manji učinak na mikrotvrdoću svjetlom polimerizirajućih materijala.
Zaprimljen: 30. rujna 2018. Prihvaćen: 1. prosinca 2018.

Adresa za dopisivanje prof. dr. sc. Katica Prskalo, dr. med. dent.

Sveučilište u Zagrebu Stomatološki fakultet HR-10000 Zagreb, Gundulićeva 5 prskalo@sfzg.hr

Ključne riječi

stomatološki materijali; svjetlosna polimerizacija adheziva; staklenoionomerni cementi, testovi tvrdoće 


\section{References}

1. Davidson, CL; Mjor, IA - editors. Advances in Glass-Ionomer Cements. Chicago: Quintessence Publishing Co., 1999; p. 51-7.

2. Sidhu SK. Glass-ionomer cements restorative materials. A sticky subject? Aust Dent J. 2011 Jun;56(1 Suppl):23-30.

3. McLean JW, Nicolson JW, Wilson AD. Proposed nomenclature for glass-ionomer dental cements and related materials. Quint Int. 1994 Sep;25(9):587-9.

4. MeSH Browser [database on the Internet]. GC Corporation, Tokyo, Japan. Fuji II LC capsules liquid SDS. Retrieved online July 3, 2018. Available from: http://www.gcaustralasia.com/Upload/product/ pdf/4/MSDS-GC-Fuji-II-LC-CAPSULE-AU.pdf

5. MeSH Browser [database on the Internet]. 3M ESPE, Seefeld, Germany. Photac Fil Quick Aplicap capsules MSDS. Retrieved online November 4, 2017. Available from: https://multimedia.3m.com/ mws/mediawebserver?mwsId=SSSSSuUn_zu8100x482v5xtG5v70k17zHvu9lxtD7SSSSSS

6. MeSH Browser [database on the Internet]. GC Corporation, Tokyo, Japan. Fuji VIII capsules MSDS. Retrieved online November 4, 2017. Available from: http://cdn.gceurope.com/v1/PID/fuji8gp/sds/SDS_FUJI_VIII_GP_Capsules_HR.pdf

7. MeSH Browser [database on the Internet]. GC Corporation, Tokyo, Japan. Fuji Plus capsules MSDS. Retrieved online June 23, 2018. Available from: https://cdn.gceurope.com/v1/PID/fujiplus/sds/ SDS_Fuji_plus_Capsule_HR.pdf

8. Nicholson JW. Chemistry of glass-ionomer cements: a review. Biomater. 1998 Mar;19(6):485-94.

9. Mount GJ. Buonocore Memorial Lecture. Glass-ionomer cements: Past, present and future. Oper Dent. 1994 May-Jun;19(3):82-90.

10. Sidhu SK, Watson TF. Resin-modified glass ionomer materials. A status report for the American Journal of Dentistry. Am J Dent. 1995 Feb;8(1):59-67.

11. Berzins DW, Abey S, Costache MC, Wilkie CA, Roberts HW. Resinmodified glass-ionomer setting reaction competition. J Dent Res. 2010 Jan;89(1):82-6.

12. Yelamanchili A, Darvell BW. Network competition in a resin-mod ified glass-ionomer cement. Dent Mater. 2008 Aug;24(8):1065-9.

13. Nicholson JW, Anstice HM. The physical chemistry of resin modi fied glass ionomers. J Mater Sci Mater Med. 1994;5(3):119-22.

14. MeSH Browser [database on the Internet]. Shofu Inc., Kyoto, Japan. Beautifil II MSDS. Retrieved online June 24, 2018. Available from: from http://www.shofu.com/shofu_images/MSDS/beautifil\%20ii.pdf

15. Ikemura K, Tay FR, Endo T, Pashley DH. A review of chemical-approach and ultramorphological studies on the development of fluoride-releasing dental adhesives comprising new pre-reacted glass ionomer (PRG) fillers. Dent Mater J. 2008 May;27(3):315-39.

16. Nicholson JW, Czarnecka B. The biocompatibility of resin-modified glass-ionomer cements for dentistry. Dent Mater. 2008 Dec;24(12):1702-8.

17. Palmer G, Anstice HM, Pearson GJ. The effect of curing regime on the release of hydroxyethyl methacrylate (HEMA) from resin-modified glass-ionomer cements. J Dent. 1999 May;27(4):303-11.

18. La Torre G, Marigo L, Pascarella GA, Rumi G. Light-emitting diodes (LED) technology applied to the photopolymerization of resin composites. Minerva Stomatol. 2003 May;52(5):193-200.

19. Yap AU, Soh MS. Curing efficacy of a new generation high-power LED lamp. Oper Dent. 2005 Nov-Dec;30(6):758-63.

20. Rueggeberg FA. State-of-the-art: Dental photocuring - A review. Dent Mater. 2011 Jan;27(1):39-52.

21. Cefaly DF, de Mello LLCP, Wang L, Lauris JRP, D’ Alpino PHP. Effect of light curing unit on resin-modified glass-ionomer cements: a microhardness assessment. J Appl Oral Sci. 2009 MayJun;17(3):150-4.
22. Mobarak E, Elsayad I, Ibrahim M, El-Badrawy W. Effect of LED light-curing on the relative hardness of tooth-colored restorative materials. Oper Dent. 2009 Jan-Feb;34(1):65-71.

23. Alpöz RA, Ertuḡrul F, Cogulu D, Ak AT, Tanoḡlu M, Kaya E. Effect of light curing method and exposure time on mechanical properties of resin based dental materials. Eur J Dent. 2008 Jan;2:37-42.

24. Parisay I, Bahrololomi Z, Ghafournia M, Solaimani AA, Boruziniat A. The effects of Exposure Times and Light Curing Sources on Surface Micro-Hardness of a Resin Modified Glass Ionomer. J Dent Mater Tech. 2014;3(2):77-81.

25. Zaazou MH, Nagi SM. Effect of light curing on micro-hardness of resin-modified versus conventional glass-ionomer restoration as function of depth and time. J Appl Sci Res. 2013;9(8):5277-82.

26. Ellakuria J, Triana R, Mínguez N, Soler I, Ibaseta G, Maza J, et al. Effect of one-year storage on the surface microhardness of resinmodified versus conventional glass-ionomer cements. Dent Mater. 2003 Jun;19(4):286-90.

27. Magni E, Ferrari M, Hickel R, Ilie N. Evaluation of the mechanical properties of dental adhesives and glass-ionomer cements. Clin Oral Investig. 2010 Feb;14(1):79-87.

28. Swift EJ Jr, Pawlus MA, Vargas MA, Fortin D. Depth of cure of resin modified glass ionomers. Dent Mater. 1995 May;11(3):196-200.

29. Roberts HW, Berzins DW, Charlton DG. Hardness of three resin-modified glass-ionomer restorative materials as a function of depth and time. J Esthet Restor Dent. 2009;21(4):262-74.

30. Yap AUJ. Post-irradiation hardness of resin-modified glass ionomer cements and a polyacid-modified composite resin. J Mater Sci Mater Med. 1997 Jul;8(7):413-6.

31. Sever I, Klaric E, Tarle Z. Accounting for measurement reliability to improve the quality of inference in dental microhardness research: a worked example. Clin Oral Invest. 2016 Jul;20(6):11439.

32. Soh MS, Yap AU. Influence of curing modes on crosslink density in polymer structures. J Dent. 2004 May;32(4):321-6.

33. da Silva EM, Poskus LT, Guimaräes JG. Influence of light-polymerization modes on degree of conversion and mechanical properties of resin composites: comparative analysis between a hybrid and nanofilled composite. Oper Dent. 2008 May-Jun;33(3):287-93.

34. Yap AU, Wang X, Wu X, Chung SM. Comparative hardness and modulus of tooth-colored restoratives: a depth-sensing microindentation study. Biomaterials. 2004 May;25(11):2179-85.

35. Harlow JE, Sullivan B, Shortall AC, Labrie D, Price RB. Characterizing the output settings of dental curing lights. Journal of Dentistry. 2016 Jan;44:20-6.

36. Spajic J, Prskalo K, Sariri K, Par M, Panduric V, Demoli N. Dimensional Changes of Glass Ionomers and a Giomer during the Setting Time. Acta stomatol Croat. 2018;52(4):298-306.

37. Roberts HW, Berzins DW. Early Reaction Kinetics of Contemporary Glass-Ionomer Restorative Materials. J Adhes Dent. 2015 Feb;17(1):67-75.

38. Young AM. FTIR investigation of polymerization and polyacid neutralization kinetics in resin-modified glass-ionomer dental cements. Biomaterials. 2002 Aug;23(15):3289-95.

39. Bala O, Arisu HD, Yikilgan I, Arslan S, Gullu A. Evaluations of surface roughness and hardness of different glass ionomer cements. Eur J Dent. 2012 Jan;6(1):79-86.

40. Xie D, Brantley WA, Culbertson, Wang G. Mechanical properties and microstructures of glass-ionomer cements. Dent Mater. 2000 Mar;16(2):129-38.

41. Kakaboura A, Eliades G, Palaghias G. An FTIR study on the setting mechanism of resin-modified glass ionomer restoratives. Dent Mater. 1996 May;12(3):173-8. 